ARTICLE

\title{
Van Hove singularity in the magnon spectrum of the antiferromagnetic quantum honeycomb lattice
}

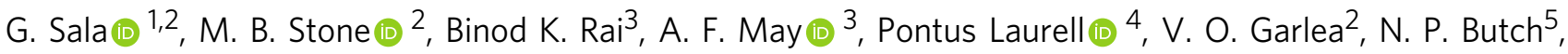

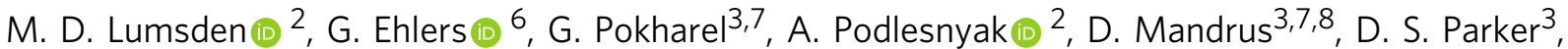 \\ S. Okamoto ${ }^{3}{ }^{3}$, Gábor B. Halász ${ }^{3} \&$ A. D. Christianson (10 ${ }^{3 凶}$
}

In quantum magnets, magnetic moments fluctuate heavily and are strongly entangled with each other, a fundamental distinction from classical magnetism. Here, with inelastic neutron scattering measurements, we probe the spin correlations of the honeycomb lattice quantum magnet $\mathrm{YbCl}_{3}$. A linear spin wave theory with a single Heisenberg interaction on the honeycomb lattice, including both transverse and longitudinal channels of the neutron response, reproduces all of the key features in the spectrum. In particular, we identify a Van Hove singularity, a clearly observable sharp feature within a continuum response. The demonstration of such a Van Hove singularity in a two-magnon continuum is important as a confirmation of broadly held notions of continua in quantum magnetism and additionally because analogous features in two-spinon continua could be used to distinguish quantum spin liquids from merely disordered systems. These results establish $\mathrm{YbCl}_{3}$ as a benchmark material for quantum magnetism on the honeycomb lattice.

\footnotetext{
${ }^{1}$ Spallation Neutron Source, Second Target Station, Oak Ridge National Laboratory, Oak Ridge, TN 37831, USA. ${ }^{2}$ Neutron Scattering Division, Oak Ridge National Laboratory, Oak Ridge, TN 37831, USA. ${ }^{3}$ Materials Science \& Technology Division, Oak Ridge National Laboratory, Oak Ridge, TN 37831, USA. ${ }^{4}$ Center for Nanophase Materials Sciences, Oak Ridge National Laboratory, Oak Ridge, TN 37831, USA. ${ }^{5}$ NIST Center for Neutron Research, National Institute of Standards and Technology, Gaithersburg, MD 20899, USA. ${ }^{6}$ Neutron Technologies Division, Oak Ridge National Laboratory, Oak Ridge, TN 37831, USA. 7 Department of Physics \& Astronomy, University of Tennessee, Knoxville, TN 37996, USA. ${ }^{8}$ Department of Materials Science \& Engineering, University of Tennessee, Knoxville, TN 37996, USA. ${ }^{凶}$ email: christiansad@ornl.gov
} 
T he honeycomb lattice decorated with interacting spins is a particularly fascinating structural motif for the generation of collective quantum behavior. This bipartite lattice geometry has the minimum coordination number of three for a lattice in two dimensions. When the interactions between the spins are strongly anisotropic, as is the case for a growing number of Kitaev materials $s^{1-22}$, the result is strongly frustrating interactions and, hence, the honeycomb lattice is presently thought of as one of the primary contenders to host quantum spin liquids. In the opposite limit of isotropic spin interactions, frustrated quantum magnetism can arise through the competition of nearest neighbor and nextnearest neighbor interactions ${ }^{23-37}$. Indeed, most honeycomb lattice materials studied thus far require the addition of further neighbor interactions to explain the underlying physical behavior ${ }^{6,8,38-47}$. Such materials, with a complicated phase diagram as a function of first, second, and third nearest-neighbor interactions, have been fertile ground for exploration.

On the other hand, finding an example of honeycomb lattice magnetism where nearest-neighbor Heisenberg interactions are dominant would provide an important benchmark for studying quantum magnetism in two dimensions. In this instance, due to the bipartite geometry of the honeycomb lattice, the Heisenberg exchange interactions are not frustrated and a Néel ground state is expected ${ }^{25,26,48}$ at zero temperature. However, long-range order at finite temperature is prohibited by the Mermin-Wagner theorem as long as there are no anisotropic or interlayer interactions. Despite the lack of frustration, in this case, the low connectivity of the honeycomb lattice indicates that collective quantum effects are likely to be observable. Experimental realizations of the ideal honeycomb lattice Heisenberg model (HLHM) are thus attractive as a means of testing fundamental concepts of collective quantum behavior.

For example, quantum effects connected to two-magnon continua, such as magnon decays and renormalizations, have been predicted for a range of two-dimensional lattices ${ }^{49}$, including the square $^{50,51}$, triangular ${ }^{52,53}$, and honeycomb ${ }^{54}$ lattices. However, while the Van Hove singularities of the two-magnon continua have a crucial role in these quantum effects, the Van Hove singularities themselves have not been experimentally observed despite extensive studies of square lattice $e^{55-57}$ and triangular lattice ${ }^{58-60}$ quantum magnets. More generally, it is important to distinguish quantum effects that arise entirely due to the honeycomb geometry from those that also require other sources, such as frustration or anisotropic interactions. A further motivation is the identification of a model system where the energy scale of the spin-spin interactions is modest enough to allow the quantum properties to be studied as a function of a relevant tuning parameter, for example, an applied magnetic field ${ }^{54}$.

Here we focus on the nearly ideal honeycomb lattice material $\mathrm{YbCl}_{3}$ as a potential model quantum magnet in the unfustrated limit. The arrangement of the $\mathrm{Yb}^{3+}$ ions is illustrated in Fig. 1. While $\mathrm{YbCl}_{3}$ is formally monoclinic (space group $\mathrm{C12} / \mathrm{ml}$ ), there is only a very modest distortion $(<0.5 \%$ difference between $\mathrm{Yb}-\mathrm{Yb}$ nearest-neighbor distances) from the ideal honeycomb lattice geometry in the $a b$ planes ${ }^{61}$. $\mathrm{YbCl}_{3}$ has been proposed as a candidate for Kitaev physics ${ }^{62,63}$, but other studies suggest that $\mathrm{YbCl}_{3}$ is likely to exist in the Heisenberg limit ${ }^{64}$. Thus, a key question concerning the physical behavior of $\mathrm{YbCl}_{3}$ is the nature of the spin interactions and the manifestation of collective quantum effects. Experimental studies thus far have found a broad signature in the specific heat peaked at $1.8 \mathrm{~K}$ that comprises $\sim 99.8 \%$ of the entropy of $R \ln (2)$ expected for the ground state doublet ${ }^{62}$. At $T=0.6 \mathrm{~K}$, a weak anomaly in the specific heat is observed. The local crystallographic environment results in easy plane anisotropy of the $\mathrm{Yb}^{3+}$ magnetic moments ${ }^{61}$. Finally, the polycrystalline averaged magnetic excitation spectrum of $\mathrm{YbCl}_{3}{ }^{61}$

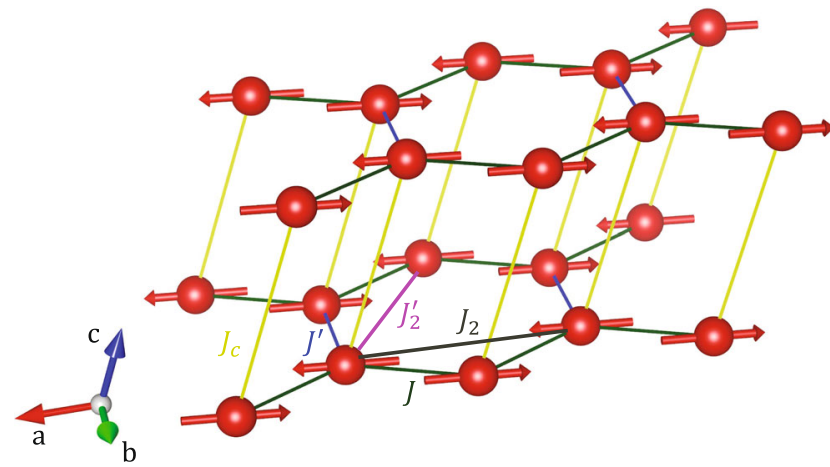

Fig. 1 Crystal and magnetic structure of $\mathrm{YbCl}_{3}$. The monoclinic crystal structure (space group $\mathrm{C} 12 / \mathrm{m} 1$ ) and ordered spin configuration (magnetic space group $\mathrm{C2}^{\prime} / \mathrm{m}$ ) of $\mathrm{YbCl}_{3}$. The monoclinic structure with $a=6.729 \AA$, $b=11.614 \AA, c=6.313 \AA$, and $\beta=110.6^{\circ}$ (at $T=10 \mathrm{~K}$ ) contains nearly ideal honeycomb lattices of $\mathrm{Yb}^{3+}$ ions (red spheres) ${ }^{61}$. Within each honeycomb lattice, the $\mathrm{Yb}^{3+}$ sites have nearest-neighbor distances of $3.884 \AA$ and $3.867 \AA$ for the exchanges $J$ and $J^{\prime}$, respectively, and next-nearest-neighbor distances of $6.729 \AA$ and $6.711 \AA$ for the exchanges $J_{2}$ and $J_{2}^{\prime}$, respectively. The resulting three bond angles for the honeycomb lattice are $120^{\circ}, 119.97^{\circ}$ and $119.97^{\circ}$. The distance between the honeycomb planes is $6.313 \AA$, corresponding to an interlayer exchange $J_{c}$. For the ideal model (see Eq. (1)), we consider $J=J^{\prime}$ and $J_{2}=J_{2}^{\prime}=J_{c}=0$. The spins are

antiferromagnetically aligned within each honeycomb plane and point primarily along the $a$ axis with a small tilt of $5(3)^{\circ}$ along the $c$ axis, as described in the text.

is rather different from that of the prototype Kitaev material $\mathrm{RuCl}_{3}{ }^{17}$, suggesting that a different set of interactions govern the physical behavior of $\mathrm{YbCl}_{3}$.

In this paper, we study $\mathrm{YbCl}_{3}$ with high-resolution inelastic neutron scattering (INS) measurements of single crystals. In addition to a conventional spin-wave (single-magnon) mode, these measurements show a sharp Van Hove singularity (VHS) within a broad two-magnon continuum that originates from longitudinal (quantum) spin fluctuations. Linear spin-wave theory with a single Heisenberg interaction on the honeycomb lattice reproduces all features of the data, demonstrating the strong quantum and almost perfectly two-dimensional character of $\mathrm{YbCl}_{3}$. Additional support for these conclusions is presented through polarized neutron diffraction and specific heat capacity measurements in conjunction with microcanonical thermal pure quantum state (mTPQ) calculations. Together, these results demonstrate that $\mathrm{YbCl}_{3}$ is an ideal model system of a twodimensional quantum magnet without frustrated or anisotropic interactions. Being considerably hard to find, such model systems are crucial in quantum magnetism as they enable the controlled experimental investigation of collective quantum behavior. Moreover, on a conceptual level, the observation of a sharp feature within a continuum response demonstrates the coherent origin of the continuum. Therefore, our results for the twomagnon continuum are proof of the principle that similar features within two-spinon continua could be utilized for more unambiguous detection of fractionalized quantum magnets, such as quantum spin liquids.

\section{Results}

Inelastic neutron scattering data. We first examine the lowenergy magnetic excitation spectra of $\mathrm{YbCl}_{3}$ at $0.24 \mathrm{~K}$. Figures $2 a-c$ and $3 a-e$ show the INS spectra as a function of energy, $\hbar \omega$, and wave vector, $\mathbf{Q}$, transfer. Figure $2 \mathrm{a}-\mathrm{f}$ is plotted as the product of the intensity and the energy transfer to emphasize higher energy features in the spectrum. The spectra contain three 

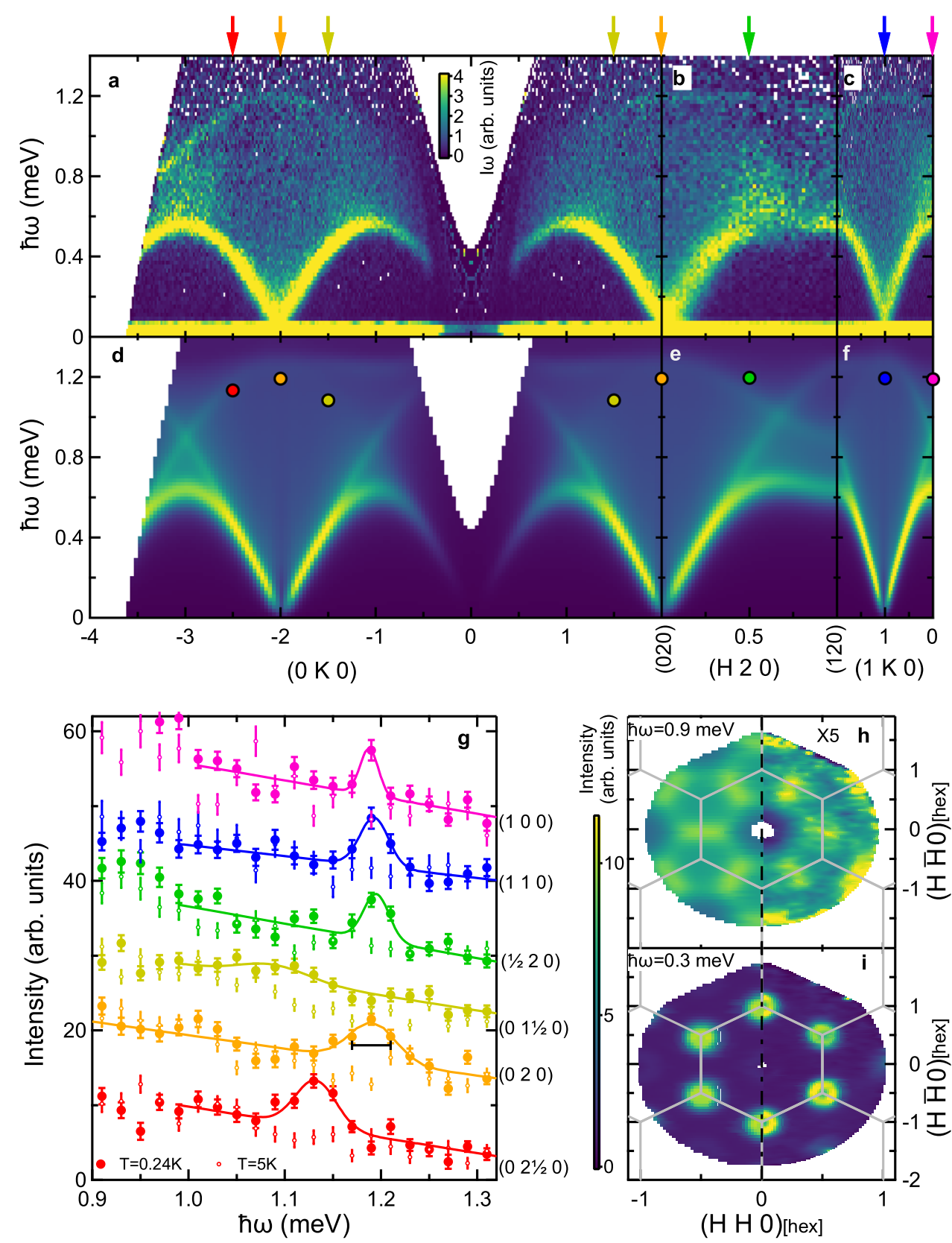

Fig. 2 Inelastic neutron scattering data and linear spin-wave theory calculations. a-c Inelastic neutron scattering data measured at $T=0.24 \mathrm{~K}$ using the CNCS instrument. $\mathbf{d}-\mathbf{f}$ Linear spin-wave calculations including transverse and longitudinal channels for the ideal model (see Eq. (1)) with a fitted value of $J=0.421(5) \mathrm{meV}$ along high symmetry directions in the (HKO) plane. Panels a-f are plotted as the product of intensity and energy transfer ( $\hbar \omega)$. The $\mathrm{Yb}^{3+}$ magnetic form factor is included in the calculations. $\mathbf{g}$ Intensity as a function of $\hbar \omega$ through the $V$ an Hove singularities at several wave vectors for $T=0.24$ $\mathrm{K}(T=5 \mathrm{~K})$, marked by solid (open) points. Solid lines are Gaussian lineshapes with a sloping background. The horizontal bar represents the energy resolution at $\hbar \omega=1.19 \mathrm{meV}$. The color of the data in ( $\mathbf{g}$ ) corresponds to the wave vector indicated by the colored arrow at the top of (a)-(c). Peak positions of the Gaussian lineshapes in ( $\mathbf{g}$ ) are shown as solid circles in (d)-(f). h-i Calculated (left) and measured (right) scattering intensity for $\hbar \omega=0.9$ meV (h) and $0.3 \mathrm{meV}$ (i). The data and calculation in (h) have been scaled by a factor of 5 to be on the same intensity scale as (i). Gray lines indicate high symmetry directions of the Brillouin zone. Wave vector transfers are shown in the reciprocal space of the monoclinic lattice in reciprocal lattice units for (a)-(g) and projected into a hexagonal lattice in (h)-(i). Data in (a)-(c) and (g) have been averaged about the $\mathrm{H}=\mathrm{O}$ and $\mathrm{K}=\mathrm{O}$ axes. Data in $(\mathbf{h})$ and $(\mathbf{i})$ have been averaged about the vertical axis.

distinct features: a component characteristic of conventional transverse spin waves $(\hbar \omega \leq 0.6 \mathrm{meV})$, a continuum or multimagnon component, and a sharper component at higher energies $(0.8 \leq \hbar \omega \leq 1.2 \mathrm{meV})$. The spin-wave mode disperses throughout the $(H K 0)$ plane with no discernible dispersion along the $(00 L)$ direction (see Fig. 3e and Fig. 5 of the Supplementary material). The lack of an observable dispersion along the $(00 \mathrm{~L})$ direction indicates that the interactions between the honeycomb planes are very weak. Interestingly, the spin-wave mode appears to be broader than the instrumental resolution. While the reason for this broadening is uncertain, it may arise due to domain formation as a result of bond disorder ${ }^{65}$, interaction with the multimagnon continuum (potentially following a renormalization of the spin-wave spectrum ${ }^{52}$ ), or even the inability to fully distinguish the overlapping continuum contribution. We also point out that the $T=3.7 \mathrm{~K}$ data in Fig. 3f illustrate a complete lack of well- 


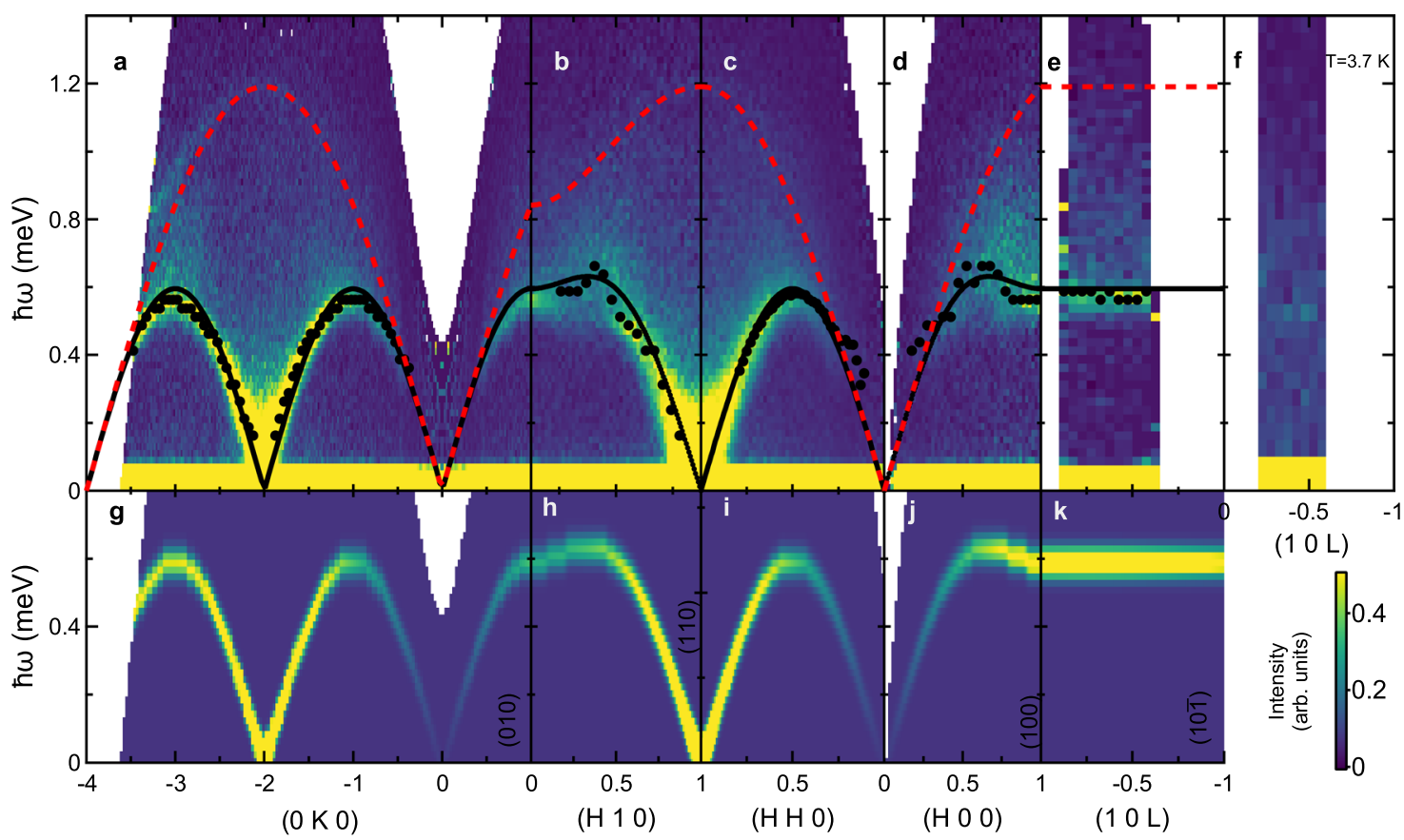

Fig. 3 Inelastic neutron scattering data and calculations for $\mathbf{Y b C l}_{\mathbf{3}}$. a-e Measured at $0.24 \mathrm{~K}$ using the CNCS instrument. $\mathbf{f}$ Measured at $3.7 \mathrm{~K}$. Black points are the locations of the absolute peak intensity at different wave vectors. The solid black line is the fitted spin-wave dispersion with a single interaction $J=$ $0.421(5) \mathrm{meV}$. The dashed red line is the calculated upper boundary of the corresponding two-magnon continuum scattering. $\mathbf{g}$-k Linear spin-wave calculations of the transverse spectrum for interactions $J=0.42(1) \mathrm{meV}, J^{\prime}=0.43(1) \mathrm{meV}$, and $J_{c}=0 \mathrm{meV}$, as described in the text. Data in (a)-(f) have been averaged about the $H=0$ and $K=0$ axes.

formed magnetic excitations at higher temperatures. Finally, an important feature of the data is the lack of an appreciable spin gap (see Supplementary material for additional details). This observation suggests that the spins do not possess a significant uniaxial anisotropy, in agreement with the crystal field ground state with easy plane anisotropy determined in ref. ${ }^{61}$.

The most unusual part of the spin excitation spectrum is the sharp feature toward the top of the broad continuum. While there is precedence for the observation of spinon and multimagnon continua in one-dimensional ${ }^{66-70}$ and two-dimensional square and triangular lattice ${ }^{55-60}$ quantum magnets, the observation of a sharp feature within such a continuum has, to the best of our knowledge, not yet been reported. This sharp multimagnon feature is explored further through constant wave vector scans in Fig. $2 \mathrm{~g}$. At energies where the sharp feature is well separated from the most intense parts of the continuum, the width is essentially limited to the calculated energy resolution of the instrument, $\mathrm{FWHM}=0.04 \mathrm{meV}$ at $\hbar \omega=1.19 \mathrm{meV}$. As will be described in more detail below, this result provides an experimental demonstration of how a sharp feature can be generated within a continuum response in a quantum magnet. Note that the hexagonal symmetry of the spin excitations is manifest in both these higher energy features and the transverse spin-wave modes at lower energies, as shown in the right sides of Fig. $2 \mathrm{~h}$ and $\mathrm{i}$, respectively.

Linear spin-wave theory. To understand the physics begetting the novel spin excitation spectrum of $\mathrm{YbCl}_{3}$, we consider a Heisenberg model on the honeycomb lattice with a single antiferromagnetic exchange interaction $J$ between nearest-neighbor $S=1 / 2$ spins

$$
H=J \sum_{\left\langle\mathbf{r}, \mathbf{r}^{\prime}\right\rangle} \vec{S}_{\mathbf{r}} \cdot \vec{S}_{\mathbf{r}^{\prime}}=J \sum_{\left\langle\mathbf{r}, \mathbf{r}^{\prime}\right\rangle}\left[S_{\mathbf{r}}^{z} S_{\mathbf{r}^{\prime}}^{z}+\frac{1}{2}\left(S_{\mathbf{r}}^{+} S_{\mathbf{r}^{\prime}}^{-}+S_{\mathbf{r}}^{-} S_{\mathbf{r}^{\prime}}^{+}\right)\right] .
$$

On the bipartite honeycomb lattice, the ground state $|0\rangle$ of this Heisenberg Hamiltonian $H$ is the antiferromagnetic Néel state $^{25,26,48}$. Assuming without loss of generality that the spins are parallel to the $z$ direction, the transverse and the longitudinal components of the dynamical spin structure factor are

$$
\begin{aligned}
\mathcal{S}_{ \pm}(\mathbf{q}, \omega)= & \frac{1}{4 \pi N} \sum_{\mathbf{r}, \mathbf{r}^{\prime}} \int_{-\infty}^{+\infty} \mathrm{dt} \mathrm{e}^{\mathrm{i} \omega \mathrm{t}-\mathrm{iq} \cdot\left(\mathbf{r}^{\prime}-\mathbf{r}\right)} \\
& {\left[g_{x}^{2}\left\langle 0\left|S_{\mathbf{r}^{\prime}}^{x}(t) S_{\mathbf{r}}^{x}(0)\right| 0\right\rangle+g_{y}^{2}\left\langle 0\left|S_{\mathbf{r}^{\prime}}^{y}(t) S_{\mathbf{r}}^{y}(0)\right| 0\right\rangle\right], } \\
\mathcal{S}_{z z}(\mathbf{q}, \omega)= & \frac{g_{z}^{2}}{4 \pi N} \sum_{\mathbf{r}, \mathbf{r}^{\prime}} \int_{-\infty}^{+\infty} \mathrm{dt} \mathrm{e}^{\mathrm{i} \omega \mathrm{t}-\mathrm{i} \mathbf{q} \cdot\left(\mathbf{r}^{\prime}-\mathbf{r}\right)}\left\langle 0\left|\mathrm{~S}_{\mathbf{r}^{\prime}}^{\mathrm{z}}(\mathrm{t}) \mathrm{S}_{\mathbf{r}}^{\mathrm{z}}(0)\right| 0\right\rangle,
\end{aligned}
$$

respectively, where $g_{x, y, z}$ are appropriate $g$ factors. In linear spinwave theory, the Hamiltonian in Eq. (1) is expanded up to quadratic order in Holstein-Primakoff bosons to obtain an analytically tractable approximation (see Supplementary material for additional details). The dynamical spin structure factors in Eq. (2) are then computed by expanding the spins up to the lowest nontrivial order in the same Holstein-Primakoff bosons, which can be identified as magnon excitations. For the transverse component, expansion of the spins up to linear order gives rise to a sharp single-magnon (spin wave) contribution

$$
\mathcal{S}_{ \pm}(\mathbf{q}, \omega)=\frac{\left(g_{x}^{2}+g_{y}^{2}\right)\left(1-\left|\lambda_{\mathbf{q}}\right| \cos \vartheta_{\mathbf{q}}\right)}{4 \sqrt{1-\left|\lambda_{\mathbf{q}}\right|^{2}}} \delta\left(\omega-\varepsilon_{\mathbf{q}}\right),
$$

corresponding to the spin-wave dispersion

$$
\omega=\varepsilon_{\mathbf{q}}=\frac{3 J}{2} \sqrt{1-\left|\lambda_{\mathbf{q}}\right|^{2}}, \quad \lambda_{\mathbf{q}}=\frac{1}{3} \sum_{j=1}^{3} e^{i \mathbf{q} \cdot \mathbf{r}_{j}},
$$

where $e^{i \vartheta_{\mathbf{q}}}=\lambda_{\mathbf{q}} /\left|\lambda_{\mathbf{q}}\right|$, and $\mathbf{r}_{1,2,3}$ are the three bond vectors connecting nearest-neighbor sites on the honeycomb lattice. For the longitudinal component, the spins must be expanded up to 
quadratic order to get a nontrivial inelastic contribution

$$
\mathcal{S}_{z z}(\mathbf{q}, \omega)=\frac{g_{z}^{2}}{4 N} \sum_{\mathbf{k}} \frac{1-\sqrt{1-\left|\lambda_{\mathbf{k}}\right|^{2}} \sqrt{1-\left|\lambda_{\mathbf{q}-\mathbf{k}}\right|^{2}}-\left|\lambda_{\mathbf{k}}\right|\left|\lambda_{\mathbf{q}-\mathbf{k}}\right| \cos \left(\vartheta_{\mathbf{k}}+\vartheta_{\mathbf{q}-\mathbf{k}}\right)}{\sqrt{1-\left|\lambda_{\mathbf{k}}\right|^{2}} \sqrt{1-\left|\lambda_{\mathbf{q}-\mathbf{k}}\right|^{2}}} \delta\left(\omega-\varepsilon_{\mathbf{k}}-\varepsilon_{\mathbf{q}-\mathbf{k}}\right) .
$$

This two-magnon contribution gives a broad continuum over a finite energy range for each momentum $\mathbf{q}$ because the energy transfer, $\omega=\varepsilon_{\mathbf{k}}+\varepsilon_{\mathbf{q}-\mathbf{k}}$, depends on the momenta $\mathbf{k}$ and $\mathbf{q}-\mathbf{k}$ of the individual magnons. We note that, in linear spin-wave theory, the staggered magnetic moment of the Néel state is only $\approx 48 \%$ of its classical value on the honeycomb lattice, in comparison to $\approx$ $61 \%$ on the square lattice ${ }^{71}$. Such a large reduction of the magnetic moment indicates that quantum fluctuations are strong due to the low coordination number of the honeycomb lattice ${ }^{72}$.

\section{Discussion}

Comparison between data and model. The HLHM in Eq. (1) reproduces the experimental data for the transverse spin-wave mode, the broad continuum, and the sharp feature toward the top of the continuum. We first note that, due to the summation over the momentum $\mathbf{k}$, the contribution from the two-magnon states in Eq. (5) results in a broad continuum of scattering. To determine the Heisenberg exchange $J$, we consider the transverse component of the data. Due to the large scattering intensity of the continuum, we compare the calculated dispersion to the overall maxima in the scattering intensity as a function of $\mathbf{q}$ (solid points in Fig. 3a-e). Comparing these values for points restricted to the (HK0) plane yields a Heisenberg exchange of $J=0.421(5) \mathrm{meV}$, shown as a solid line in Fig. 3a-e. Moreover, we directly compare the data along the $(0 K 0),(H 20)$, and $(1 K 0)$ directions to the numerical evaluation of Eqs. (3) and (5) convolved with a Gaussian approximation to the instrumental energy and wave vector resolution functions while also including the spherical approximation for the $\mathrm{Yb}^{3+}$ magnetic form factor and an additive background term. The resulting spectra are shown in Fig. $2 \mathrm{~d}-\mathrm{f}$ and $\mathrm{h}-\mathrm{i}$; the continuum response and the sharp feature within the continuum are reproduced exceptionally well. (see Supplementary material for additional comparisons between the data and the model.) We remark that the excellent agreement between the experimental data and the ideal HLHM is also in accordance with the prediction of ref. ${ }^{64}$.

The sharp feature toward the top of the continuum is a particularly interesting aspect of the spectrum that, to the best of our knowledge, has not been previously observed in a quantum magnet. In the model, such a sharp feature appears within the two-magnon continuum due to a VHS in the joint density of states $49,50,52-54$. Indeed, on the level of pure kinematics (i.e., ignoring any matrix element effects), the longitudinal twomagnon response in Eq. (5) is proportional to the joint density of states, $\hat{g}_{\mathbf{q}}(\omega)=\sum_{\mathbf{k}} \delta\left(\omega-\varepsilon_{\mathbf{k}}-\varepsilon_{\mathbf{q}-\mathbf{k}}\right)$, at each momentum $\mathbf{q}$, which corresponds to the joint band dispersion $\hat{\varepsilon}_{\mathbf{q}}(\mathbf{k})=\varepsilon_{\mathbf{k}}+$ $\varepsilon_{\mathbf{q}-\mathbf{k}}$ as a function of the individual magnon momentum $\mathbf{k}$. Being a two-dimensional band dispersion, $\hat{\varepsilon}_{\mathbf{q}}(\mathbf{k})$ has a saddle-point VHS which gives a logarithmic divergence in the density of states $\hat{g}_{\mathbf{q}}(\omega)$ and, thus, in the longitudinal spin response. Physically, the VHS is a specific energy transfer $\omega$ that can create many distinct magnon pairs with a fixed total momentum $\mathbf{q}$ but different individual momenta $\mathbf{k}$ and $\mathbf{q}-\mathbf{k}$. The coalescence of such distinct scattering processes is analogous to the coalescence of light rays giving rise to caustic features in ray optics (see Fig. 4). Therefore, using this analogy, the VHS can also be understood as a caustic feature in the longitudinal spin dynamics. We emphasize that the observation of a VHS is direct evidence for strong quantum fluctuations in $\mathrm{YbCl}_{3}$ (because the VHS appears in the longitudinal spin response) as well as the two-dimensional nature of its quantum magnetism (because significant interlayer exchange would smear out the VHS).

Upon close examination, the analytic model does not fully capture the intensity and dispersion of the VHS over the entire zone, as can be seen in Fig. 2a-f. By plotting the fitted peak positions of the VHS from Fig. $2 \mathrm{~g}$ on the calculated spectra in Fig. 2d-f, we notice that there are differences between the calculated and the observed VHS energies near the (100) and $\left(\frac{1}{2} 20\right)$ wave vectors $(\approx 0.2 \mathrm{meV})$. These energy differences may arise from a renormalization of the two-magnon continuum due to magnon interactions neglected in linear spin-wave theory. Also, we point out that domain formation due to bond disorder ${ }^{65}$ could, in principle, play a role in partially smearing out the VHS and thus explaining why certain portions of the predicted VHS are absent from the experimental data.

We now explore the potential importance of additional exchange interactions through comparison of the experimental spin-wave dispersion, including points measured along the $L$ direction, to calculations using the SpinW software ${ }^{73}$. Since there are very small differences in the bond lengths within the honeycomb layers of $\mathrm{YbCl}_{3}$, as described in Fig. 1, we label two of the three nearestneighbor exchanges as $J$ for the $d=3.884 \AA$ bonds and the third one as $J^{\prime}$ for the $d=3.867 \AA$ bond. The numerical comparison yields $J=0.42(1) \mathrm{meV}$ and $J^{\prime}=0.43(1) \mathrm{meV}$, while $J_{c}$ refines to a value below the detection limit of $0.016 \mathrm{meV}$ for the measurements reported here (see Supplementary material for additional details). The resulting cross-section is shown in Fig. $3 \mathrm{~g}-\mathrm{k}$. The numerically determined $J$ and $J^{\prime}$ are indistinguishable from each other and close to the value determined by a comparison to the analytical model. The spin-wave modes from linear spin-wave theory accurately reproduce the dispersion and intensity distribution of this portion of the measured spectrum (Fig. 3g-k). We also attempted to include next-nearest-neighbor exchange interactions within the plane of the honeycomb lattice; the best fit values of $J_{2}$ and $J_{2}^{\prime}$ are three orders of magnitude smaller than $J$ and zero within the error bars of the refined value (see Supplementary material for additional details).

Magnetic moment and Néel temperature. To further check the validity of the ideal HLHM, we have performed polarized neutron diffraction measurements at $T=0.3 \mathrm{~K}$ (see Supplementary material for additional details). We observe an antiferromagnetic spin arrangement with a well-defined staggered magnetic moment. By symmetry, this moment can either lie in the ac plane or point along the $b$ axis, i.e., a linear combination of the two directions is not allowed. Our measurements reveal that the magnitude of the ordered moment is $1.06(4) \mu_{B}$ and that the moment points primarily along the $a$ axis with only a small deviation of $5(3)^{\circ}$ along the $c$ axis. The expected moment from the ground state crystal field doublet is 2.24 (5) $\mu_{B}{ }^{61}$. The value of the ordered moment is thus $\approx 47 \%$ of the fully polarized moment, which is in excellent agreement with the value of $\approx 48 \%$ obtained from linear spin-wave theory and the value of $\approx 54 \%$ determined by more accurate techniques ${ }^{72,74-77}$. We emphasize, however, that these theoretical values are extremely sensitive to any interlayer exchange $J_{c}$. For example, if we include $\left|J_{c} / J\right| \approx 4 \%$, corresponding to the detection limit from the INS response (see above), the ordered moment in linear spin wave theory already increases from $\approx 48 \%$ to $\approx 63 \%$. Therefore, the small value of the experimental moment indicates that the actual value of $J_{c}$ is significantly below the INS detection limit and further confirms our conclusion that the magnetism in $\mathrm{YbCl}_{3}$ is two dimensional to a very good approximation.

The same conclusion is demonstrated even more strikingly by the very small value of the Néel temperature, $T_{N} \approx 0.6 \mathrm{~K}^{62}$. Theoretically, for a quasi-two-dimensional Heisenberg magnet, 

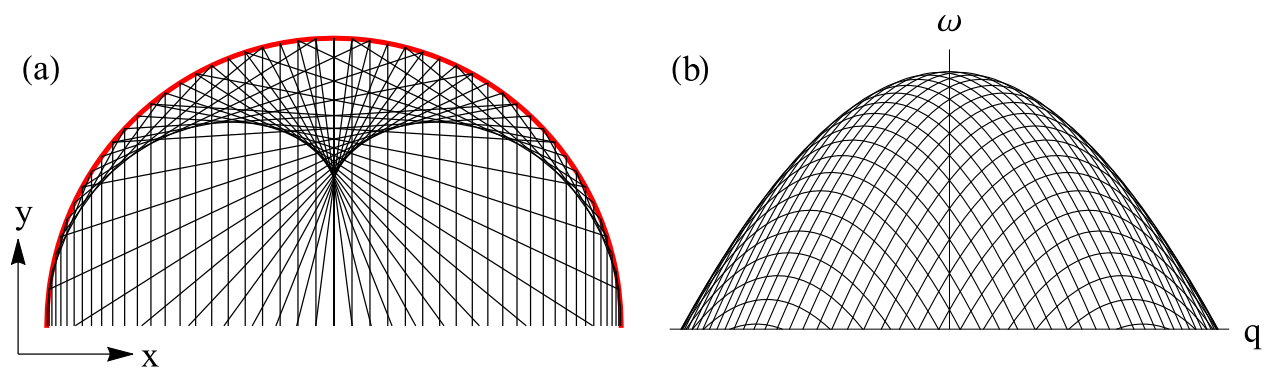

Fig. 4 Alternative understanding of Van Hove singularities as caustic features in the collective spin dynamics. a Caustic features in ray optics. Parallel light rays (black lines) enter an optical system at different positions. When these light rays reflecting from a circular mirror (red line) coalesce, they give rise to caustic features in real space. $\mathbf{b}$ Caustic features in a spin response. The two-magnon continuum can be understood as a sum of sharp contributions, $\omega=\varepsilon_{\mathbf{k}}+\varepsilon_{\mathbf{q}-\mathbf{k}}$, each corresponding to a fixed momentum $\mathbf{k}$ of the first magnon. When these sharp contributions (black lines) coalesce, they give rise to caustic features in the two-magnon continuum. Note that the spin response shown here is for a one-dimensional model system; for the two-dimensional system in consideration, the caustic features appear inside the continuum (not at its edge) and are weaker as they correspond to logarithmic (rather than square-root) singularities.

the Néel temperature is approximately given by $T_{N}=4 \pi \rho_{s} /$ $\log \left|\mathrm{J} / \mathrm{J}_{\mathrm{c}}\right|$, where $\rho_{s}$ is the spin stiffness of the purely twodimensional system. At this temperature, $T=T_{N}$, the individual interlayer exchanges $J_{c}$ add constructively within an area of linear size $\xi \sim \exp \left(2 \pi \rho_{s} / T\right)^{78,79}$ to produce an effective interlayer exchange on the order of the intralayer exchange $J$. Taking $\rho_{s} \approx$ $0.101 \mathrm{~J}$ from the literature ${ }^{76,77}$ and $J \approx 0.42 \mathrm{meV}$ from the neutron scattering data, the relative interlayer exchange is then estimated to be $\left|J_{c} / J\right| \sim \exp \left(-4 \pi \rho_{s} / T_{N}\right) \approx 3 \times 10^{-5}$. This value is three orders of magnitude smaller than the detection limit from the INS response and indicates that the magnetism in $\mathrm{YbCl}_{3}$ is as close to two-dimensional as in the canonical cuprate antiferromagnet $\mathrm{La}_{2} \mathrm{CuO}_{4}{ }^{80}$. Moreover, the specific ordering pattern below $T_{N}$ signals the presence of very small anisotropic interactions on top of the dominant Heisenberg exchange $J$. Since these anisotropic spin interactions should also contribute to $T_{N}$, the interlayer exchange may be even smaller than our estimate. We finally remark that the application of a small magnetic field should, in principle, cause the ordering transition to sharpen and the Néel temperature to increase ${ }^{79}$. While there are experimental indications for such behavior in the specific heat ${ }^{62}$, more detailed studies of the field dependence would be very important to confirm not only this prediction but also further predictions of interesting quantum effects at larger magnetic fields ${ }^{54}$.

Heat capacity calculations. Specific heat capacity measurements provide an additional means of examining the HLHM in $\mathrm{YbCl}_{3}$. The experimental specific heat and the entropy of $\mathrm{YbCl}_{3}$ for $0.5 \mathrm{~K}$ $<T<8 \mathrm{~K}$ are shown in Fig. $5 \mathrm{a}$ and b. Between $T=0.5 \mathrm{~K}$ and $T=$ $8 \mathrm{~K}$, nearly all of the entropy, $R \ln (2)$, for the ground state doublet has been recovered by the system with only a very small contribution in the region of the transition to long-range magnetic order $^{62}$. We use mTPQ calculations ${ }^{81}$, as implemented in the $\mathcal{H} \Phi$ library $^{82}$, for a cluster size of 32 spin $\frac{1}{2}$ elements to calculate the heat capacity as a function of the reduced temperature $T / J$ (see Supplementary material for additional details). The results for the HLHM with $J=0.42 \mathrm{meV}$, obtained by fitting the INS data, are shown in Fig. 5a and b. The overall shape is in reasonable agreement with the data, but a somewhat improved comparison is found by using $J=0.32 \mathrm{meV}$. The discrepancy may be due to quantum corrections, such as renormalizations due to magnon interactions ${ }^{51,52}$, that are captured by the mTPQ calculations but neglected in linear spin wave theory.

We have used INS to investigate the collective magnetic excitation spectrum of $\mathrm{YbCl}_{3}$. In addition to a conventional

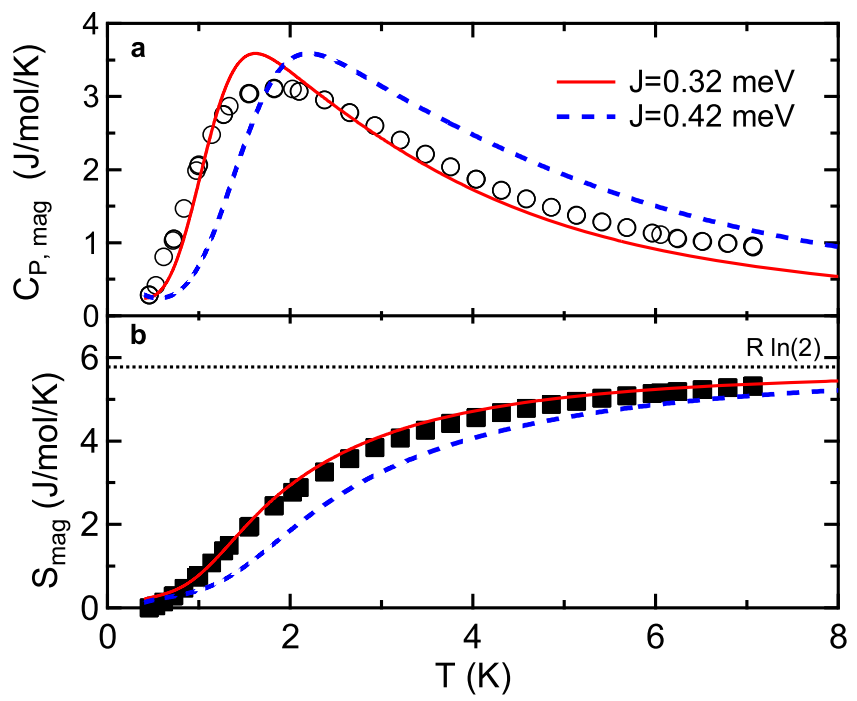

Fig. 5 Magnetic specific heat capacity, entropy, and mTPQ calculations for $\mathbf{Y b C l}_{\mathbf{3}}$. Specific heat capacity (a) and entropy (b) for $\mathrm{YbCl}_{3}$. The solid red line is the best fit calculation using $\mathrm{mTPQ}$ as described in the text. The dashed blue line is the calculated specific heat using mTPQ with the value $J=0.42 \mathrm{meV}$ obtained by fitting the INS data.

transverse spin-wave (single-magnon) mode, there is a longitudinal two-magnon continuum harboring a sharp VHS. These components are all reproduced by linear spin-wave theory with a single nearest-neighbor Heisenberg interaction on the honeycomb lattice. The sharp VHS is observable due to the almost perfectly two-dimensional quantum magnetism in $\mathrm{YbCl}_{3}$, which is further reflected in a strongly reduced ordered moment and a very small ordering temperature. Our results establish $\mathrm{YbCl}_{3}$ as an ideal model system to investigate the collective quantum behavior of the honeycomb antiferromagnet in the unfrustrated limit. Finally, we point out that the observation of a VHS in a two-magnon continuum provides a strong indication that a similar observation in the two-spinon spectrum of a twodimensional quantum spin liquid ${ }^{83,84}$ is experimentally feasible. Such an observation in a quantum spin liquid would be important in ruling out competing sources of a continuum response, such as quenched disorder or overdamped magnons, and could provide a more unambiguous signature of a long-range-entangled quantum state. 


\section{Methods}

Single crystals of $\mathrm{YbCl}_{3}$ were grown using the Bridgman technique in evacuated silica ampoules (see Supplementary material and ref. ${ }^{85}$ for further details). INS measurements were performed with the cold neutron chopper spectrometer $(\mathrm{CNCS})^{86}$ at the Spallation Neutron Source at Oak Ridge National Laboratory. Additional measurements were made with the disk chopper spectrometer (DCS) at NIST (see Supplementary material for additional details). The CNCS measurements were performed with a $0.625 \mathrm{~g}$ sample oriented with the $(H K 0)$ scattering plane horizontal using $2.49 \mathrm{meV}$ incident energy, $E_{i}$, neutrons in the high flux configuration of the instrument. To minimize the effects of the modest neutron absorption cross section of $\mathrm{Yb}$ and $\mathrm{Cl}$, the sample used at CNCS was constructed of a stack of plates cut to dimensions of $3.2 \mathrm{~mm}$ by $3.4 \mathrm{~mm}$. The polarized HYSPEC measurements were performed using the same sample as the CNCS experiment. We employed 3D polarization analysis, i.e., we measured the $P_{x}, P_{y}$, and $P_{z}$ spin-flip and non-spin flip channels. Additional details are provided in the Supplementary material.

\section{Data availability}

The authors declare that the data supporting the findings of this study are available within the article and its Supplementary Information files. Additional data are available from the corresponding author upon reasonable request.

\section{Code availability}

All numerical codes used in this paper are available from the corresponding author upon reasonable request.

Received: 16 October 2020; Accepted: 24 November 2020;

Published online: 08 January 2021

\section{References}

1. Kitaev, A. Anyons in an exactly solved model and beyond. Ann. Phys. 321, 2-111 (2006).

2. Takagi, H., Takayama, T., Jackeli, G., Khaliullin, G. \& Nagler, S. E. Concept and realization of Kitaev quantum spin liquids. Nat. Rev. Phys. 1, 264-280 (2019).

3. Motome, Y. \& Nasu, J. Hunting majorana fermions in Kitaev magnets. J. Phys. Soc. Jpn. 89, 012002 (2020).

4. Jackeli, G. \& Khaliullin, G. Mott insulators in the strong spin-orbit coupling limit: from Heisenberg to a quantum compass and Kitaev models. Phys. Rev. Lett. 102, 017205 (2009).

5. Chaloupka, J. c. v., Jackeli, G. \& Khaliullin, G. Kitaev-Heisenberg model on a honeycomb lattice: possible exotic phases in iridium oxides $\mathrm{A}_{2} \mathrm{IrO}_{3}$. Phys. Rev. Lett. 105, 027204 (2010).

6. Singh, Y. \& Gegenwart, P. Antiferromagnetic Mott insulating state in single crystals of the honeycomb lattice material $\mathrm{Na}_{2} \mathrm{IrO}_{3}$. Phys. Rev. B. 82, 064412 (2010).

7. Singh, Y. et al. Relevance of the Heisenberg-Kitaev model for the honeycomb lattice iridates $A_{2} \mathrm{IrO}_{3}$. Phys. Rev. Lett. 108, 127203 (2012).

8. Ye, F. et al. Direct evidence of a zigzag spin-chain structure in the honeycomb lattice: a neutron and $\mathrm{x}$-ray diffraction investigation of single-crystal $\mathrm{Na}_{2} \mathrm{IrO}_{3}$. Phys. Rev. B. 85, 180403 (2012).

9. Hwan Chun, S. et al. Direct evidence for dominant bond-directional interactions in a honeycomb lattice iridate $\mathrm{Na}_{2} \mathrm{IrO}_{3}$. Nat. Phys. 11, 462-466 (2015).

10. Williams, S. C. et al. Incommensurate counterrotating magnetic order stabilized by Kitaev interactions in the layered honeycomb $\alpha-\mathrm{Li}_{2} \mathrm{IrO}_{3}$. Phys. Rev. B. 93, 195158 (2016).

11. Kitagawa, K. et al. A spin-orbital-entangled quantum liquid on a honeycomb lattice. Nature. 554, 341-345 (2018).

12. Plumb, K. W. et al. $\alpha-\mathrm{RuCl}_{3}$ : a spin-orbit assisted mott insulator on a honeycomb lattice. Phys. Rev. B. 90, 041112 (2014)

13. Sears, J. A. et al. Magnetic order in $\alpha-\mathrm{RuCl}_{3}$ : a honeycomb-lattice quantum magnet with strong spin-orbit coupling. Phys. Rev. B. 91, 144420 (2015).

14. Majumder, M. et al. Anisotropic $\mathrm{Ru}^{3+} 4 d^{5}$ magnetism in the $\alpha-\mathrm{RuCl}_{3}$ honeycomb system: susceptibility, specific heat, and zero-field NMR. Phys. Rev. B. 91, 180401 (2015).

15. Johnson, R. D. et al. Monoclinic crystal structure of $\alpha-\mathrm{RuCl}_{3}$ and the zigzag antiferromagnetic ground state. Phys. Rev. B. 92, 235119 (2015).

16. Sandilands, L. J. et al. Spin-orbit excitations and electronic structure of the putative Kitaev magnet $\alpha-\mathrm{RuCl}_{3}$. Phys. Rev. B. 93, 075144 (2016).

17. Banerjee, A. et al. Proximate Kitaev quantum spin liquid behaviour in a honeycomb magnet. Nat. Mater. 15, 733-740 (2016).

18. Baek, S.-H. et al. Evidence for a field-induced quantum spin liquid in $\alpha-\mathrm{RuCl}_{3}$. Phys. Rev. Lett. 119, 037201 (2017).

19. Do, S.-H. et al. Majorana fermions in the Kitaev quantum spin system $\alpha$ $\mathrm{RuCl}_{3}$. Nat. Phys. 13, 1079 (2017).

20. Banerjee, A. et al. Excitations in the field-induced quantum spin liquid state of $\alpha-\mathrm{RuCl}_{3}$. npj Quantum Mater. 3, 8 (2018).
21. Hentrich, R. et al. Unusual phonon heat transport in $\alpha-\mathrm{RuCl}_{3}$ : strong spin-phonon scattering and field-induced spin gap. Phys. Rev. Lett. 120, 117204 (2018).

22. Kasahara, Y. et al. Majorana quantization and half-integer thermal quantum hall effect in a Kitaev spin liquid. Nature. 559, 227-231 (2018).

23. Katsura, S., Ide, T. \& Morita, T. The ground states of the classical Heisenberg and planar models on the triangular and plane hexagonal lattices. J. Stat. Phys 42, 381-404 (1986).

24. Takano, K. Spin-gap phase of a quantum spin system on a honeycomb lattice. Phys. Rev. B. 74, 140402 (2006).

25. Bishop, R. F. \& Li, P. H. Y. Complete phase diagram of the spin $-\frac{1}{2} J_{1}-J_{2}-J_{3}$ model (with $J_{3}=J_{2}$ ) on the honeycomb lattice. Phys. Rev. B. 85, 155135 (2012)

26. Mulder, A., Ganesh, R., Capriotti, L. \& Paramekanti, A. Spiral order by disorder and lattice nematic order in a frustrated Heisenberg antiferromagnet on the honeycomb lattice. Phys. Rev. B. 81, 214419 (2010).

27. Mosadeq, H., Shahbazi, F. \& Jafari, S. A. Plaquette valence bond ordering in a $J_{1}-J_{2}$ Heisenberg antiferromagnet on a honeycomb lattice. J. Phys.: Condens. Matter. 23, 226006 (2011).

28. Clark, B. K., Abanin, D. A. \& Sondhi, S. L. Nature of the spin liquid state of the Hubbard model on a honeycomb lattice. Phys. Rev. Lett. 107, 087204 (2011).

29. Ganesh, R., Sheng, D. N., Kim, Y.-J. \& Paramekanti, A. Quantum paramagnetic ground states on the honeycomb lattice and field-induced Néel order. Phys. Rev. B. 83, 144414 (2011).

30. Zhang, H. \& Lamas, C. A. Exotic disordered phases in the quantum $J_{1}-J_{2}$ model on the honeycomb lattice. Phys. Rev. B. 87, 024415 (2013).

31. Rosales, H. D., Cabra, D. C., Lamas, C. A., Pujol, P. \& Zhitomirsky, M. E. Broken discrete symmetries in a frustrated honeycomb antiferromagnet. Phys. Rev. B. 87, 104402 (2013)

32. Zhu, Z., Huse, D. A. \& White, S. R. Unexpected $z$-direction Ising antiferromagnetic order in a frustrated spin-1/2 $J_{1}-J_{2} X Y$ model on the honeycomb lattice. Phys. Rev. Lett. 111, 257201 (2013).

33. Rastelli, E., Tassi, A. \& Reatto, L. Non-simple magnetic order for simple Hamiltonians. Physica B+C. 97, 1-24 (1979).

34. Fouet, J. B., Sindzingre, P. \& Lhuillier, C. An investigation of the quantum $J_{1}$ $J_{2}-J_{3}$ model on the honeycomb lattice. Eur. Phys. J. B. 20, 241-254 (2001).

35. Albuquerque, A. F. et al. Phase diagram of a frustrated quantum antiferromagnet on the honeycomb lattice: magnetic order versus valencebond crystal formation. Phys. Rev. B. 84, 024406 (2011).

36. Rehn, J., Sen, A., Damle, K. \& Moessner, R. Classical spin liquid on the maximally frustrated honeycomb lattice. Phys. Rev. Lett. 117, 167201 (2016)

37. Mezzacapo, F. \& Boninsegni, M. Ground-state phase diagram of the quantum $J_{1}-J_{2}$ model on the honeycomb lattice. Phys. Rev. B. 85, 060402 (2012).

38. Matsuda, M. et al. Frustrated magnetic interactions in an $S=3 / 2$ bilayer honeycomb lattice compound $\mathrm{Bi}_{3} \mathrm{Mn}_{4} \mathrm{O}_{12}\left(\mathrm{NO}_{3}\right)$. Phys. Rev. B. 100, 134430 (2019).

39. Regnault, L.-P., Boullier, C. \& Lorenzo, J. Polarized-neutron investigation of magnetic ordering and spin dynamics in $\mathrm{BaCo}_{2}\left(\mathrm{AsO}_{4}\right)_{2}$ frustrated honeycomb-lattice magnet. Heliyon. 4, e00507 (2018).

40. Smirnova, O. et al. Synthesis, crystal structure, and magnetic properties of $\mathrm{Bi}_{3} \mathrm{Mn}_{4} \mathrm{O}_{12}\left(\mathrm{NO}_{3}\right)$ oxynitrate comprising $S=3 / 2$ honeycomb lattice. J. Am Chem. Soc. 131, 8313-8317 (2009).

41. Karunadasa, $H$. et al. Honeycombs of triangles and magnetic frustration in $\mathrm{Sr}_{2} \mathrm{O}_{4}(L=\mathrm{Gd}$, Dy, Ho, Er, Tm, and Yb). Phys. Rev. B. 71, 144414 (2005)

42. Lefrançois, E. et al. Magnetic properties of the honeycomb oxide $\mathrm{Na}_{2} \mathrm{Co}_{2} \mathrm{TeO}_{6}$. Phys. Rev. B. 94, 214416 (2016).

43. Nair, H. S. et al. Short-range order in the quantum XXZ honeycomb lattice material $\mathrm{BaCo}_{2}\left(\mathrm{PO}_{4}\right)_{2}$. Phys. Rev. B. 97, 134409 (2018).

44. Lançon, D., Ewings, R. A., Guidi, T., Formisano, F. \& Wildes, A. R. Magnetic exchange parameters and anisotropy of the quasi-two-dimensional antiferromagnet $\mathrm{NiPS}_{3}$. Phys. Rev. B. 98, 134414 (2018).

45. McNally, D. E. et al. $\mathrm{CaMn}_{2} \mathrm{Sb}_{2}$ : spin waves on a frustrated antiferromagnetic honeycomb lattice. Phys. Rev. B. 91, 180407 (2015).

46. Zvereva, E. A. et al. Zigzag antiferromagnetic quantum ground state in monoclinic honeycomb lattice antimonates $A_{3} \mathrm{Ni}_{2} \mathrm{Sbo}_{6}(\mathrm{~A}=\mathrm{Li}, \mathrm{Na})$. Phys. Rev. B. 92, 144401 (2015).

47. Choi, S. K. et al. Spin waves and revised crystal structure of honeycomb iridate $\mathrm{Na}_{2} \mathrm{IrO}_{3}$. Phys. Rev. Lett. 108, 127204 (2012).

48. Cabra, D. C., Lamas, C. A. \& Rosales, H. D. Quantum disordered phase on the frustrated honeycomb lattice. Phys. Rev. B. 83, 094506 (2011).

49. Zhitomirsky, M. E. \& Chernyshev, A. L. Colloquium: spontaneous magnon decays. Rev. Mod. Phys. 85, 219-242 (2013).

50. Mourigal, M., Zhitomirsky, M. E. \& Chernyshev, A. L. Field-induced decay dynamics in square-lattice antiferromagnets. Phys. Rev. B. 82, 144402 (2010).

51. Powalski, M., Schmidt, K. P. \& Uhrig, G. S. Mutually attracting spin waves in the square-lattice quantum antiferromagnet. SciPost Phys. 4, 1 (2018).

52. Chernyshev, A. L. \& Zhitomirsky, M. E. Spin waves in a triangular lattice antiferromagnet: decays, spectrum renormalization, and singularities. Phys Rev. B. 79, 144416 (2009).

53. Mourigal, M., Fuhrman, W. T., Chernyshev, A. L. \& Zhitomirsky, M. E. Dynamical structure factor of the triangular-lattice antiferromagnet. Phys. Rev B. 88, 094407 (2013). 
54. Maksimov, P. A. \& Chernyshev, A. L. Field-induced dynamical properties of the XXZ model on a honeycomb lattice. Phys. Rev. B. 93, 014418 (2016).

55. Christensen, N. B. et al. Quantum dynamics and entanglement of spins on a square lattice. Proc. Natl Acad. Sci. USA 104, 15264-15269 (2007).

56. Tsyrulin, $\mathrm{N}$. et al. Two-dimensional square-lattice $s=\frac{1}{2}$ antiferromagnet $\mathrm{Cu}(p z)_{2}\left(\mathrm{ClO}_{4}\right)_{2}$. Phys. Rev. B. 81, 134409 (2010).

57. Dalla Piazza, B. et al. Fractional excitations in the square-lattice quantum antiferromagnet. Nat. Phys. 11, 62-68 (2015).

58. Ito, $\mathrm{S}$. et al. Structure of the magnetic excitations in the spin-1/2 triangularlattice Heisenberg antiferromagnet $\mathrm{Ba}_{3} \mathrm{CoSb}_{2} \mathrm{O}_{9}$. Nat. Commun. 8, 235 (2017).

59. Kamiya, Y. et al. The nature of spin excitations in the one-third magnetization plateau phase of $\mathrm{Ba}_{3} \mathrm{CoSb}_{2} \mathrm{O}_{9}$. Nat. Commun. 9, 2666 (2018).

60. Macdougal, D. et al. Avoided quasiparticle decay and enhanced excitation continuum in the spin $-\frac{1}{2}$ near-Heisenberg triangular antiferromagnet $\mathrm{Ba}_{3} \mathrm{CoSb}_{2} \mathrm{O}_{9}$. Phys. Rev. B 102, 064421 (2020).

61. Sala, G. et al. Crystal field splitting, local anisotropy, and low-energy excitations in the quantum magnet $\mathrm{YbCl}_{3}$. Phys. Rev. B. 100, 180406 (2019).

62. Xing, J. et al. Néel-type antiferromagnetic order and magnetic field-temperature phase diagram in the spin $-\frac{1}{2}$ rare-earth honeycomb compound $\mathrm{YbCl}_{3}$. Phys. Rev. B. 102, 014427 (2020).

63. Luo, Z.-X. \& Chen, G. Honeycomb rare-earth magnets with anisotropic exchange interactions. SciPost Phys. Core 3, 4 (2020).

64. Rau, J. G. \& Gingras, M. J. P. Frustration and anisotropic exchange in ytterbium magnets with edge-shared octahedra. Phys. Rev. B 98, 054408 (2018).

65. Parker, E. \& Balents, L. Finite-temperature behavior of a classical spin-orbitcoupled model for $\mathrm{YbMgGaO}_{4}$ with and without bond disorder. Phys. Rev. B. 97, 184413 (2018).

66. Tennant, D. A., Cowley, R. A., Nagler, S. E. \& Tsvelik, A. M. Measurement of the spin-excitation continuum in one-dimensional $\mathrm{KCuF}_{3}$ using neutron scattering. Phys. Rev. B. 52, 13368-13380 (1995).

67. Tennant, D. A. et al. Neutron scattering study of two-magnon states in the quantum magnet copper nitrate. Phys. Rev. B. 67, 054414 (2003).

68. Stone, M. B., Zaliznyak, I. A., Hong, T., Broholm, C. L. \& Reich, D. H. Quasiparticle breakdown in a quantum spin liquid. Nature. 440, 187-190 (2006).

69. Gannon, W. J. et al. Spinon confinement and a sharp longitudinal mode in $\mathrm{Yb}_{2} \mathrm{Pt}_{2} \mathrm{~Pb}$ in magnetic fields. Nat. Commun. 10, 1123 (2019).

70. Wu, L. S. et al. Tomonaga-luttinger liquid behavior and spinon confinement in $\mathrm{YbAlO}_{3}$. Nat. Commun. 10, 698 (2019).

71. Lüscher, A. \& Läuchli, A. M. Exact diagonalization study of the antiferromagnetic spin- $\frac{1}{2}$ Heisenberg model on the square lattice in a magnetic field. Phys. Rev. B. 79, 195102 (2009)

72. Weihong, Z., Oitmaa, J. \& Hamer, C. J. Second-order spin-wave results for the quantum XXZ and XY models with anisotropy. Phys. Rev. B. 44, 11869-11881 (1991).

73. Toth, S. \& Lake, B. Linear spin wave theory for single-q incommensurate magnetic structures. J. Phys.: Condens. Matter. 27, 166002 (2015).

74. Oitmaa, J., Hamer, C. J. \& Weihong, Z. Quantum magnets on the honeycomb and triangular lattices at $T=0$. Phys. Rev. B. 45, 9834-9841 (1992).

75. Castro, E. V., Peres, N. M. R., Beach, K. S. D. \& Sandvik, A. W. Site dilution of quantum spins in the honeycomb lattice. Phys. Rev. B. 73, 054422 (2006).

76. Jiang, F. J. High precision determination of the low-energy constants for the two-dimensional quantum Heisenberg model on the honeycomb lattice. Eur. Phys. J. B. 85, 402 (2012).

77. Bishop, R. F., Li, P. H. Y., Götze, O., Richter, J. \& Campbell, C. E. Frustrated Heisenberg antiferromagnet on the honeycomb lattice: Spin gap and lowenergy parameters. Phys. Rev. B. 92, 224434 (2015).

78. Chakravarty, S., Halperin, B. I. \& Nelson, D. R. Two-dimensional quantum Heisenberg antiferromagnet at low temperatures. Phys. Rev. B. 39, 2344-2371 (1989).

79. Sengupta, P. et al. Nonmonotonic field dependence of the Néel temperature in the quasi-two-dimensional magnet $\left[\mathrm{Cu}\left(\mathrm{HF}_{2}\right)\left(\mathrm{pyz}_{2}\right] \mathrm{BF}_{4}\right.$. Phys. Rev. B. 79, 060409 (2009)

80. Majlis, N., Selzer, S. \& Strinati, G. C. Dimensional crossover in the magnetic properties of highly anisotropic antiferromagnets. Phys. Rev. B. 45, 7872-7881 (1992).

81. Sugiura, S. \& Shimizu, A. Thermal pure quantum states at finite temperature. Phys. Rev. Lett. 108, 240401 (2012)

82. Kawamura, M. et al. Quantum lattice model solver $\mathcal{H} \phi$. Comp. Phys. Commun. 217, 180 (2017).

83. Halász, G. B., Perkins, N. B. \& van den Brink, J. Resonant inelastic x-ray scattering response of the Kitaev honeycomb model. Phys. Rev. Lett. 117, 127203 (2016).

84. Halász, G. B., Kourtis, S., Knolle, J. \& Perkins, N. B. Observing spin fractionalization in the Kitaev spin liquid via temperature evolution of indirect resonant inelastic X-ray scattering. Phys. Rev. B. 99, 184417 (2019).
85. May, A. F., Yan, J. \& McGuire, M. A. A practical guide for crystal growth of van der Waals layered materials. J. Appl. Phys. 128, 051101 (2020).

86. Ehlers, G., Podlesnyak, A. A., Niedziela, J. L., Iverson, E. B. \& Sokol, P. E. The new cold neutron chopper spectrometer at the spallation neutron source: design and performance. Rev. Sci. Instrum. 82, 085108 (2011).

\section{Acknowledgements}

We thank C.D. Batista, N.B. Perkins, and S. Do for useful discussions. This work was supported by the U.S. Department of Energy, Office of Science, Basic Energy Sciences, Materials Sciences and Engineering Division. This research used resources at the Spallation Neutron Source and the High Flux Isotope Reactor, a Department of Energy (DOE) Office of Science User Facility operated by Oak Ridge National Laboratory (ORNL). The research by P.L. and S.O. was supported by the Scientific Discovery through Advanced Computing (SciDAC) program funded by the US Department of Energy, Office of Science, Advanced Scientific Computing Research and Basic Energy Sciences, Division of Materials Sciences and Engineering. This research used resources of the Compute and Data Environment for Science (CADES) at the Oak Ridge National Laboratory, which is supported by the Office of Science of the U.S. Department of Energy under Contract No. DE-AC05-00OR22725. G.P. was partially supported by the Gordon and Betty Moore Foundation's EPiQS Initiative through Grant GBMF4416. The work of G.B.H. was supported by the Laboratory Directed Research and Development Program of Oak Ridge National Laboratory, managed by UT-Battelle, LLC, for the US Department of Energy. This manuscript has been authored by UT-Battelle, LLC under Contract No DE-AC05-00OR22725 with the U.S. Department of Energy. The United States Government retains and the publisher, by accepting the article for publication, acknowledges that the United States Government retains a non-exclusive, paid-up, irrevocable, worldwide license to publish or reproduce the published form of this manuscript, or allow others to do so, for United States Government purposes. The Department of Energy will provide public access to these results of federally sponsored research in accordance with the DOE Public Access Plan (http://energy.gov/downloads/doe-public-access-plan).

\section{Author contributions}

A.D.C. conceived and managed the project. G.S., M.B.S., B.K.R., V.O.G., N.P.B., A.P., G.P., and A.D.C. performed the neutron scattering experiments. G.S. and M.B.S. performed the fits to the neutron scattering data. B.K.R. and A.F.M. synthesized the samples A.F.M. performed heat capacity measurements. G.B.H. performed the spin wave calculations. P.L. performed the mTPQ calculations. D.S.P. and S.O. provided theoretical input. G.S., M.B.S., A.F.M., G.B.H., and A.D.C. wrote the paper with input from all coauthors.

\section{Competing interests}

The authors declare no competing interests.

\section{Additional information}

Supplementary information is available for this paper at https://doi.org/10.1038/s41467020-20335-5.

Correspondence and requests for materials should be addressed to A.D.C.

Peer review information Nature Communications thanks the anonymous reviewer(s) for their contribution to the peer review of this work. Peer reviewer reports are available.

Reprints and permission information is available at http://www.nature.com/reprints

Publisher's note Springer Nature remains neutral with regard to jurisdictional claims in published maps and institutional affiliations.

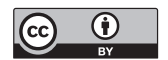

Open Access This article is licensed under a Creative Commons Attribution 4.0 International License, which permits use, sharing, adaptation, distribution and reproduction in any medium or format, as long as you give appropriate credit to the original author(s) and the source, provide a link to the Creative Commons license, and indicate if changes were made. The images or other third party material in this article are included in the article's Creative Commons license, unless indicated otherwise in a credit line to the material. If material is not included in the article's Creative Commons license and your intended use is not permitted by statutory regulation or exceeds the permitted use, you will need to obtain permission directly from the copyright holder. To view a copy of this license, visit http://creativecommons.org/ licenses/by/4.0/.

This is a U.S. government work and not under copyright protection in the U.S.; foreign copyright protection may apply 2021 